\title{
Supraclavicular lymph node dissection with radiotherapy versus radiotherapy alone for operable breast cancer with synchronous ipsilateral supraclavicular lymph node metastases: a real-world cohort study
}

\author{
Xiang $\mathrm{Ai}^{1}$, Minghao Wang ${ }^{1}$, Junyan $\mathrm{Li}^{2}$, Ying $\mathrm{Hu}^{1}$, Lingmi Hou ${ }^{3}$, Xiaodong Zheng ${ }^{4}$, Yuzhao Yan ${ }^{1}$, Qinwen \\ Pan $^{1}$, Yuting Jin ${ }^{1}$, Wei Liu ${ }^{1}$, Xuanni Tan ${ }^{1}$, Yuan Tian ${ }^{1}$, Yi Zhang ${ }^{1}$, Peng Tang ${ }^{1}$, Jun Jiang ${ }^{1}$ \\ ${ }^{1}$ Breast Disease Center, Southwest Hospital, The Army Military Medical University, Chongqing 400038, China; ${ }^{2}$ Department of Breast Surgery, \\ People's Hospital of DeYang City, Deyang 618000, China; ${ }^{3}$ Department of Thyroid Breast Surgery, First Affiliated Hospital, North Sichuan Medical \\ College, Nanchong 637000, China; ${ }^{4}$ Department of Breast Surgery, Chongqing University Cancer Hospital, Chongqing 40044, China \\ Contributions: (I) Conception and design: P Tang, J Jiang; (II) Administrative support: P Tang, J Jiang; (III) Provision of study materials or patients: X \\ Ai; (IV) Collection and assembly of data: X Zheng, Y Yan, Q Pan, Y Jin, W Liu, X Tan, Y Tian; (V) Data analysis and interpretation: J Li, Y Hu, L \\ Hou, Q Pan, X Tan; (VI) Manuscript writing: All authors; (VII) Final approval of manuscript: All authors. \\ Correspondence to: Jun Jiang, MD. Peng Tang, MD. Breast Disease Center, Southwest Hospital, The Army Military Medical University, 30 Gaotanyan \\ Road, Chongqing 400038, China. Email: jcbd@medmail.com.cn; tp1232000@sina.com.
}

\begin{abstract}
Background: The role of supraclavicular lymph node dissection (SCLD) in the treatment of breast cancer with ipsilateral supraclavicular lymph node metastasis (ISLM) remains controversial. We evaluated the role of SCLD in the treatment of breast cancer with ISLM and identified patients who may benefit from SCLD.

Methods: Data on patients presenting with breast cancer to the Breast Disease Center, Southwest Hospital, The Army Medical University from January 2004 and December 2017 were retrospectively screened. The median duration of follow-up was 36 months (2-175 months). 305 patients who were recently diagnosed with ISLM were eligible for the analysis.

Results: Overall, 9,236 women presented with breast cancer during the study period. Among the patients included, 146 and 159 received SCLD with radiotherapy (RT) and RT alone, respectively. Synchronous ISLM without distant metastases were present in 3.6\% cases. The 3- and 5-year overall survival (OS) and disease-free survival (DFS) rates were $79.5 \%$ and $73.9 \%$, respectively, and $67.5 \%$ and $54.8 \%$, respectively. However, SCLD with RT was not associated with superior survival on both univariate and multivariate analyses. On stratified analyses, patients with non-luminal A tumors with 4-9 positive axillary lymph nodes who underwent SCLD with RT had both superior OS (HR =5.296; 95\% CI: 1.857-15.107; P=0.001) and DFS (HR =5.331; 95\% CI: 2.348-12.108; $\mathrm{P}<0.001)$ compared with those who received RT alone.
\end{abstract}

Conclusions: SCLD may not beneficial in improving survival for unselected breast cancer patients with ISLNM. There is less of a tendency to perform SCLD in the luminal A group.

Keywords: Breast cancer; N3c; supraclavicular lymph node; supraclavicular lymph node dissection (SCLD); survival

Submitted Nov 14, 2019. Accepted for publication Feb 08, 2020.

doi: 10.21037 /gs.2020.03.09

View this article at: http://dx.doi.org/10.21037/gs.2020.03.09 


\section{Introduction}

The incidence of breast cancer with ISLM and without distant metastases at presentation is $1.3-4.3 \%(1-3)$. In 1987, the American Joint Committee on Cancer (AJCC) TNM staging system, $5^{\text {th }}$ edition, classified ISLM as M1 disease (4). Consequently, patients with ISLM were considered to have a poor prognosis and were treated with palliative intent only $(5,6)$. However, in 2001, when Brito et al. (7) reported that patients with ISLM had better outcomes than those with distant metastases, the AJCC $6^{\text {th }}$ edition re-classified ISLM as N3c disease in 2003 (8).

Since then, ISLM has been regarded as potentially curable disease with a favorable long-term prognosis if treated appropriately (9). The treatment approach changed and these patients started receiving combined aggressive treatment, including neo-adjuvant chemotherapy (NAC), surgery, adjuvant chemotherapy, radiotherapy (RT), and endocrine and molecular targeted therapy (10-14). Local treatment for ISLM usually comprises regional lymph node dissection and radical RT, which may in theory, prevent the tumor cells from spreading through lymph-vessels.

Several studies have focused on the prognosis of patients with ISLM (15-19), most were retrospective studies with small sample sizes. Currently, the evidence for the role of SCLD in the treatment of patients with ISLM from breast cancer is scarce.

In this study, we evaluated the prognostic factors associated with survival in patients with breast cancer and synchronous ISLM diagnosed on imaging. We also conducted stratified analyses to identify those who may benefit from SCLD.

\section{Methods}

\section{Identification of patients}

Data on patients presenting with breast cancer to the Breast Disease Center, Southwest Hospital, The Army Medical University between January 2004 and December 2017, were retrospectively screened. Among a total of 9,236 patients, ISLM was diagnosed in 353 by ultrasonography or positron emission tomography-computed tomography (PETCT). Suspected signs of malignancy in the supraclavicular lymph nodes on ultrasonography included the following: (I) irregular shape, (II) vague boundaries, (III) changes in internal architecture (loss of hilar architecture, presence of intranodal necrosis, and calcification), (IV) unclear cortical medullary boundaries, and (V) presence of vascularity. In clinical practice, lymph nodes are considered to be positive for metastasis when 3 or more of these suspicious signs are present. Patients with suspicious findings on ultrasound were referred for fluorodeoxyglucose-positron emission tomography/computer tomography (FDG-PET/CT) when a definite diagnosis could not be made. Lymph nodes with a higher uptake than the surrounding normal tissue, excluding physiological uptake, were considered to have metastases.

Patients who had other malignant tumors $(n=14)$, ipsilateral supraclavicular lymph node enlargement due to other reasons $(n=7)$, and those who did not undergo standardized treatment $(\mathrm{n}=27)$ were excluded from analysis (Figure 1). Finally, 305 patients with ISLM, diagnosed either by ultrasonography $(\mathrm{n}=254)$ or positron emission tomography-computed tomography (PET-CT) $(\mathrm{n}=51)$ were eligible. All included patients had been assessed at presentation by the clinical history, findings on physical examination, mammography, ultrasonography, chest computed tomography (CT), breast magnetic resonance imaging (MRI), and bone scintigraphy.

\section{Study variables}

The primary outcome of interest was survival. We evaluated the clinicopathological variables for each case, including age, tumor location (left- or right-sided, central, or by quadrant), T stage (AJCC TNM stage, $6^{\text {th }}$ edition), pathological type, ER (positivity defined as $>1 \%$ ), PR (positivity defined as $>1 \%$ ), Ki67 status, HER2 status, molecular subtype, number of involved axillary lymph nodes (surgical specimen after NAC), and infraclavicular lymph node status. The ER and PR status, Ki-67 labelling index, and HER2 status were evaluated immunohistochemically; fluorescence in situ hybridization (FISH) for the HER2 status was performed as required. Patients were classified into 4 molecular subtypes, namely, luminal A (ER and PR positive, HER2 negative, and Ki67 <14\%), luminal B (ER positive and/or PR positive and HER2 positive, or ER positive and/or PR positive and HER2 negative), HER2 (ER and $\mathrm{PR}$ negative and HER2 positive), and triple negative (ER and PR negative and HER2 negative).

\section{Treatment}

\section{Chemotherapy}

Patients received 4-8 cycles of anthracycline-based 


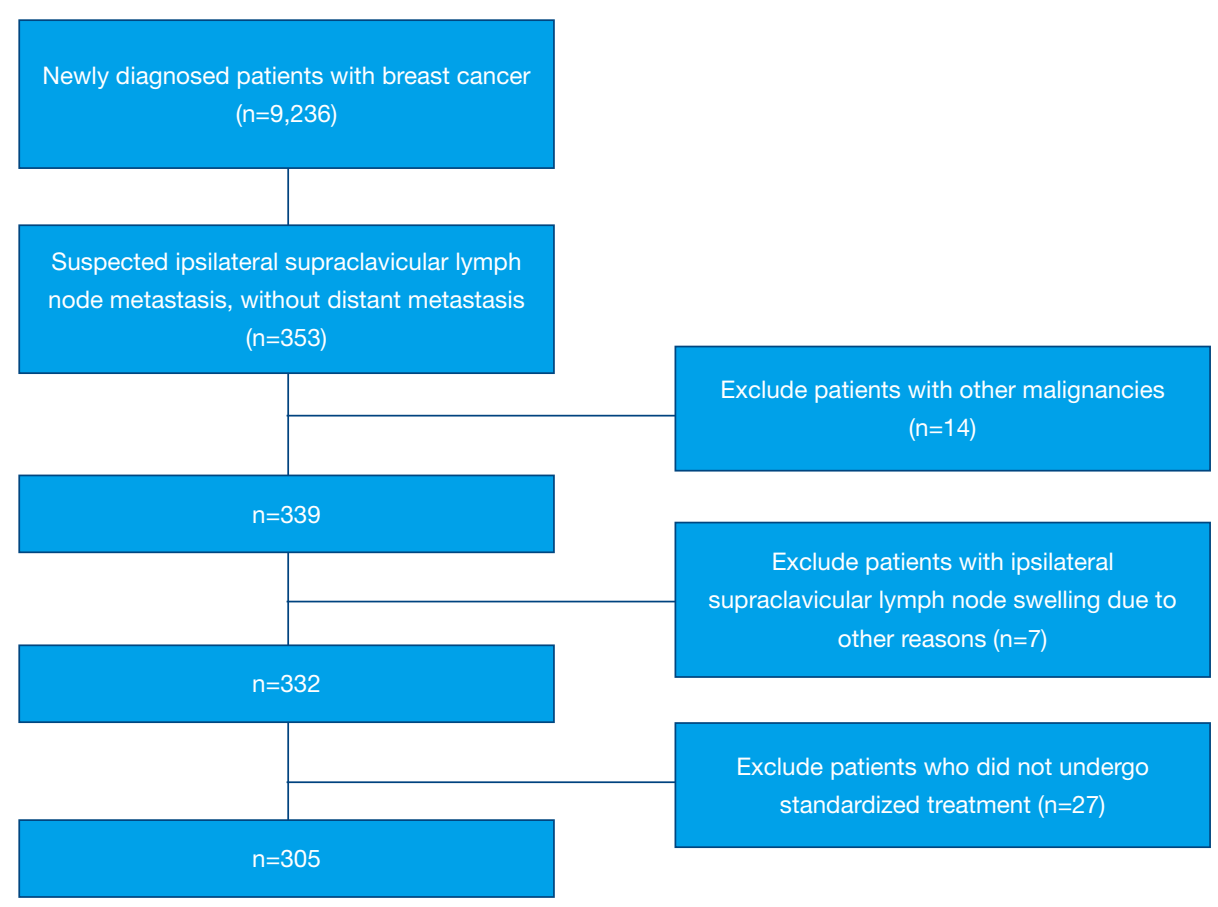

Figure 1 Diagram of study cohort selection steps.

neoadjuvant chemotherapy (NAC); the initial doses were according to the recommendations of the National Comprehensive Cancer Network (NCCN) guidelines. Dose adjustments in the protocol were individualized on a case basis. In those who received some of the courses of chemotherapy prior to surgery, the remaining courses were completed in the adjuvant setting.

\section{Surgery}

All patients underwent modified radical mastectomy after NAC; axillary lymph node dissection of levels I, II, and III was also performed. SCLD was performed depending on the findings on ultrasonography/PET-CT. The scope of SCLD usually involved a triangular region formed by the internal jugular vein (medial border), subclavian vein (lower border), and omohyoid (lateral and superior border) ( $8^{\text {th }}$ AJCC), which contains neck nodes of levels IV, VB, and part of III. Lymph nodes outside this area were also explored and suspicious enlarged lymph nodes were sent for pathological examination separately.

\section{RT}

Adjuvant regional RT included the chest wall, axilla, and the infraclavicular, supraclavicular, and internal mammary regions. The prescribed dose ranged from 46 to $50 \mathrm{~Gy}$ in 23-25 fractions.

\section{Additional adjuvant treatment}

Further adjuvant treatment was administered in accordance with the recommendations of the NCCN guidelines. Overall, 171 and 62 patients received endocrine therapy for ER-positive or PR-positive disease and trastuzumab (Herceptin) for HER2 overexpression, respectively. ERpositive or PR-positive breast cancer patients underwent postoperative endocrine therapy with tamoxifen or an aromatase inhibitor. Trastuzumab was incorporated into the adjuvant treatment and administered for 1 year after surgery.

\section{Assessment of efficacy}

The rates of events were calculated by dividing the number of first observed events during follow-up by the number of individuals from the date of surgery till the date of the last visit. Disease-free survival (DFS) was defined as the time starting from the date of surgery to relapse or death. OS was defined as the time from the date of surgery till death from any cause. The efficacy of NAC was assessed using 
the Response Evaluation Criteria in Solid Tumors criteria, which define response as follows: progressive disease (PD): a $\geq 20 \%$ increase in the total length of the baseline lesion or a new lesion; stable disease (SD): decrease in the total length of the baseline lesions but not sufficient for PR, or have increased but have not reached $\mathrm{PD}$; partial response (PR): decrease in the total length of the baseline lesions by $\geq 30 \%$; complete response (CR): disappearance of all target lesions. $\mathrm{PR}+\mathrm{CR}$ and $\mathrm{PD}+\mathrm{SD}$ were defined as valid and invalid responses, respectively.

\section{Follow-up}

Every patient was evaluated at 1, 3, 6, and 12 months after surgery for SCLD-related complications.

During this period, patients were examined every 3 to 6 months for 2 years after the surgery, every 6 to 12 months between 3 and 5 years, and once a year after 5 years.

The survival status of patients lost to follow-up for more than 1 year was obtained either over telephone from contacts or through local death registers. Follow-up visits included the evaluation of general health status, physical examination, ultrasonography of the breast and accessory lymph nodes, chest radiographs, and other imaging examinations as required.

\section{Statistical analysis}

The Pearson's chi-square test was used to compare the clinicopathologic categorical variables. Survival curves for time-to-event variables were estimated using the KaplanMeier method and were compared using log-rank tests. Factors with a $\mathrm{P}$ value $<0.1$ were considered relevant and were entered into the Cox proportional hazards regression analysis. We subsequently performed a stratified analysis to further evaluate the impact of SCLD on subsets of patients with ISLM. Patients were stratified by independent factors associated with survival. During stratified analysis, the conclusions were based on the fact that the proportions of distribution of the independent prognostic factors on multivariate analysis showed no significant difference between groups. The Pearson's Chi-square test was used to compare the proportions of the distribution. All the statistical analyses were performed using SPSS statistical software, version 25.0 (IBM Corp, Armonk, NY, USA).

\section{Results}

Data from 9,236 breast cancer patients were retrospectively screened. Among them, 305 patients were finally eligible for analysis in our study. eventually, counting in 27 patients who did not undergo standardized treatment, the rate of synchronous ISLM in this cohort was 3.6\%. Among those analyzed, 146 and 159 patients received SCLD with RT and RT alone, respectively.

\section{Clinicopathological characteristics}

The clinicopathological characteristics of this cohort are summarized in Table 1.

The median age was 48 years (range, 25-83 years); 22 (7.2\%) and $283(92.8 \%)$ patients were younger and older than 35 years of age, respectively. The primary tumor was on the left and right sides in $180(59.0 \%)$ and $125(41.0 \%)$ patients, respectively. Most of the patients $(61.0 \%)$ had primary tumors in the upper outer quadrant. The primary tumor sizes in 57 (18.7\%), 195 (63.9\%), 29 (9.5\%), and 24 (7.9\%) patients were T1, T2, T3, and T4, respectively. A total of $251(82.3 \%)$ patients had a diagnosis of invasive ductal carcinoma. The tumors in 198 (64.9\%), 166 (54.4\%), and $83(27.2 \%)$ patients tested positive for ER, PR, and HER2, respectively. The number of involved axillary nodes in 107 (35.1\%), 72 (23.6\%), 65 (21.3\%), and 61 (20.0\%) patients were $0,1-3,4-9$, and $\geq 10$, respectively. The infraclavicular lymph nodes were involved in 54 (17.7\%) patients; 146 patients (47.9\%) underwent supraclavicular lymph node dissection (SCLD) while the remaining 159 (52.1\%) did not.

The clinicopathological characteristics of the SCLD and RT cohorts are summarized in Table S1.

\section{Survival outcomes}

The median duration of follow-up in the 305 patients finally included, was 36 months (2-175 months). The $3-$ and 5 -year OS and DFS were $79.5 \%$ and $73.9 \%$, and $67.5 \%$ 
Table 1 ISLM patient clinicopathological characteristics

\begin{tabular}{|c|c|c|}
\hline \multirow{2}{*}{ Characteristic } & \multicolumn{2}{|c|}{ ISLM (n=305) } \\
\hline & No. & $\%$ \\
\hline \multicolumn{3}{|l|}{ Age (years) } \\
\hline$<35$ & 22 & 7.2 \\
\hline$\geq 35$ & 283 & 92.8 \\
\hline \multicolumn{3}{|l|}{ Tumor location } \\
\hline Left & 180 & 59.0 \\
\hline Right & 125 & 41.0 \\
\hline \multicolumn{3}{|l|}{ Tumor quadrant } \\
\hline Outer upper & 186 & 61.0 \\
\hline Outer lower & 27 & 8.9 \\
\hline Inner upper & 28 & 9.2 \\
\hline Inner lower & 17 & 5.6 \\
\hline central & 47 & 15.4 \\
\hline \multicolumn{3}{|l|}{ Primary tumor size } \\
\hline $\mathrm{T} 1$ & 57 & 18.7 \\
\hline $\mathrm{T} 2$ & 195 & 63.9 \\
\hline T3 & 29 & 9.5 \\
\hline $\mathrm{T} 4$ & 24 & 7.9 \\
\hline \multicolumn{3}{|l|}{ Histological type } \\
\hline Invasive ductal carcinoma & 251 & 82.3 \\
\hline Other types & 54 & 17.7 \\
\hline \multicolumn{3}{|l|}{ ER } \\
\hline Negative & 107 & 35.1 \\
\hline Positive & 198 & 64.9 \\
\hline \multicolumn{3}{|l|}{ PR } \\
\hline Negative & 139 & 45.6 \\
\hline Positive & 166 & 54.4 \\
\hline \multicolumn{3}{|l|}{ HER-2 } \\
\hline Negative & 222 & 72.8 \\
\hline Positive & 83 & 27.2 \\
\hline \multicolumn{3}{|l|}{ Molecular subtype } \\
\hline Luminal A & 80 & 26.2 \\
\hline Luminal B & 121 & 39.7 \\
\hline HER2-overexpressing & 40 & 13.1 \\
\hline
\end{tabular}

Table 1 (continued)
Table 1 (continued)

\begin{tabular}{|c|c|c|}
\hline \multirow{2}{*}{ Characteristic } & \multicolumn{2}{|c|}{ ISLM $(n=305)$} \\
\hline & No. & $\%$ \\
\hline TNBC & 64 & 21.0 \\
\hline \multicolumn{3}{|l|}{ Ki67 } \\
\hline$\leq 14 \%$ & 82 & 26.9 \\
\hline$>14 \%$ & 223 & 73.1 \\
\hline \multicolumn{3}{|c|}{ The number of involved axillary lymph nodes } \\
\hline 0 & 107 & 35.1 \\
\hline $1-3$ & 72 & 23.6 \\
\hline $4-9$ & 65 & 21.3 \\
\hline$\geq 10$ & 61 & 20.0 \\
\hline \multicolumn{3}{|c|}{ Involvement of infraclavicular lymph node } \\
\hline No & 251 & 82.3 \\
\hline Yes & 54 & 17.7 \\
\hline \multicolumn{3}{|l|}{ SCLD } \\
\hline No & 159 & 52.1 \\
\hline Yes & 146 & 47.9 \\
\hline \multicolumn{3}{|l|}{ NAC } \\
\hline$P D+S D$ & 119 & 39.0 \\
\hline $\mathrm{PR}+\mathrm{CR}$ & 186 & 61.0 \\
\hline \multicolumn{3}{|c|}{$\begin{array}{l}\text { ISLM, ipsilateral supraclavicular lymph node metastasis; ER, } \\
\text { estrogen receptor; PR, progestational hormone; HER-2, human } \\
\text { epidermal growth factor receptor-2; TNBC, triple negative breast } \\
\text { cancer; SCLD, supraclavicular lymph node dissection; NAC, } \\
\text { neoadjuvant chemotherapy; PD, progressive disease; SD, stable } \\
\text { disease; PR, partial disease; CR, complete disease. }\end{array}$} \\
\hline
\end{tabular}

and $54.8 \%$, respectively. The Kaplan-Meier curves are shown in Figure $2 A$. On unadjusted analysis, patients in the SCLD cohort had poorer survival; the 3 - and 5 -year OS was $69.8 \%$ and $65.8 \%$ in the SCLD cohort vs. $87.0 \%$ and $80.2 \%$ in the RT cohort. $(\mathrm{P}=0.018)$ (Figure $2 B$ ). Similar results were obtained for 3- and 5-year DFS in the SCLD and RT cohorts, which were $61.1 \%$ and $49.0 \%$, respectively, and $72.0 \%$ and $8.3 \%$, respectively; this difference was statistically significant $(\mathrm{P}=0.020)$ (Figure $2 C$ ).

\section{Univariate analysis for survival}

On univariable analysis, no significant association was noted 

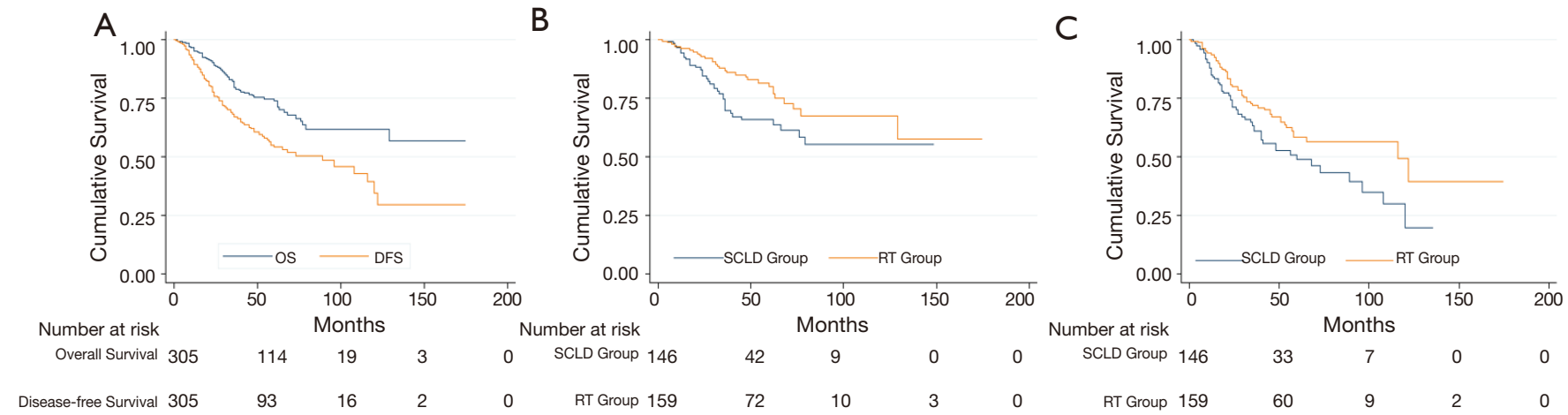

Figure 2 Kaplan-Meier curves. (A) Kaplan-Meier estimates of overall survival and disease-free survival for all patients with supraclavicular lymph node metastases; (B) Kaplan-Meier analysis comparing overall survival in patients treated with SCLD plus RT and RT alone; (C) Kaplan-Meier analysis comparing disease- free survival in patients treated with SCLD plus RT and RT alone. SCLD, supraclavicular lymph node dissection; RT, radiotherapy.

between age at diagnosis of synchronous ISLM, tumor location, tumor quadrant, primary tumor size, histological type, HER2 status, and the OS and DFS. However, negative ER status (OS: $\mathrm{P}<0.001$, DFS: $\mathrm{P}<0.001$ ), negative $\mathrm{PR}$ status (OS: $\mathrm{P}<0.001$, DFS: $\mathrm{P}<0.001$ ), Ki67 $\geq 14 \%$ (OS: $\mathrm{P}=0.025$, DFS: $\mathrm{P}=0.016)$, involvement of infraclavicular lymph nodes (OS: $\mathrm{P}<0.001$, DFS: $\mathrm{P}<0.001$ ), and SCLD (OS: $\mathrm{P}=0.018$, DFS: $\mathrm{P}=0.020)$ were associated with poor prognosis. The molecular subtype $(\mathrm{OS}: \mathrm{P}<0.001$, DFS: $\mathrm{P}<0.001)$ and number of involved axillary lymph nodes (OS: $\mathrm{P}<0.001$, DFS: $\mathrm{P}<0.001)$ were significantly associated with both, OS and DFS (Table 2).

\section{Cox multivariable model for assessment of risk factors}

After controlling for significant covariables including ER, PR, Ki67, molecular subtype, infraclavicular lymph node involvement, number of involved axillary lymph nodes, SCLD in the Cox multivariable model, upfront molecular subtype $(\mathrm{P}<0.001)$, the number of involved axillary lymph nodes $(\mathrm{P}=0.013)$ and infraclavicular lymph node involvement were independently associated with OS (HR $=0.544 ; 95 \%$ CI: $0.305-0.973 ; \mathrm{P}=0.040)$. Upfront $\mathrm{ER}(\mathrm{HR}$ $=1.871 ; 95 \%$ CI: $1.061-3.300 ; \mathrm{P}=0.030), \mathrm{PR}(\mathrm{HR}=1.826$; 95\% CI: 1.032-3.232; $\mathrm{P}=0.039)$, number of involved axillary lymph nodes $(\mathrm{P}<0.001)$, and infraclavicular lymph node involvement $(\mathrm{HR}=0.568 ; 95 \% \mathrm{CI}: 0.354-0.913 ; \mathrm{P}=0.019)$ were independently associated with DFS. Nevertheless,
SCLD was not significantly associated with OS or DFS (Table 3).

\section{Additional stratified analyses}

To identify potential differences in the benefits of SCLD in patients with variable prognosis, we compared the baseline independent prognostic factors between the SCLD and RT cohorts. The patients were initially categorized by the number of involved axillary lymph nodes. In patients with 0 positive axillary lymph nodes, SCLD demonstrated no benefits in either OS (HR: 0.361; 95\% CI: 0.126-1.033, $\mathrm{P}=0.058$; Figure 3) or DFS (HR: 0.773; 95\% CI: 0.3461.725; $\mathrm{P}=0.529 ;$ Figure 4). No significant differences in OS (HR: 0.796; 95\% CI: 0.364-1.745; $\mathrm{P}=0.570$ ) and DFS (HR: 0.767 ; 95\% CI: $0.379-1.551 ; \mathrm{P}=0.460$ ) were noted in patients with more than 10 positive axillary lymph nodes in either group. Considering the baseline independent prognostic factors in either cohort, patients with 1-3, 4-9 positive axillary lymph nodes were unbalanced, and no conclusions could be made. On further stratified analyses, we found that SCLD may offer significant benefits in patients with non-luminal A tumors and 4-9 positive axillary lymph nodes, offering superior OS (HR: 5.296; 95\% CI: 1.857-15.107; $\mathrm{P}=0.001 ;$ Figures 5,6A) and DFS (HR: 5.331; 95\% CI: 2.348-12.108; $\mathrm{P}<0.001$; Figures 6B,7) compared to RT. No other subgroup of the SCLD and RT cohorts demonstrated significant differences in OS or DFS when 
Table 2 Univariable analyses of factors associated with survival outcomes

\begin{tabular}{|c|c|c|c|c|}
\hline Characteristic & $\begin{array}{c}3-y r(5-y r) \text { OS } \\
(\%)\end{array}$ & $\mathrm{P}$ value & $\begin{array}{c}3-y r(5-y r) \text { DFS } \\
(\%)\end{array}$ & $\mathrm{P}$ value \\
\hline Age (years) & & 0.391 & & 0.240 \\
\hline$<35$ & $75(67.5)$ & & $55.4(55.4)$ & \\
\hline$\geq 35$ & $79.8(74.5)$ & & $68.0(53.7)$ & \\
\hline Tumor location & & 0.465 & & 0.130 \\
\hline Left & 76.7 (73.4) & & $63.2(49.8)$ & \\
\hline Right & $83.5(74.5)$ & & $72.6(60.6)$ & \\
\hline Tumor quadrant & & 0.946 & & 0.801 \\
\hline Outer upper & 79.7 (73.0) & & $65.6(53.1)$ & \\
\hline Outer lower & $86.4(67.3)$ & & $59.9(49.9)$ & \\
\hline Inner upper & $75.7(75.7)$ & & $68.3(44.8)$ & \\
\hline Inner lower & 76.9 (76.9) & & $67.8(45.2)$ & \\
\hline central & $78.3(78.3)$ & & $73.3(62.9)$ & \\
\hline Primary tumor size & & 0.110 & & 0.111 \\
\hline $\mathrm{T} 1$ & $85.9(83)$ & & $72.2(62.1)$ & \\
\hline $\mathrm{T} 2$ & $79.8(73.5)$ & & $68.3(55.8)$ & \\
\hline T3 & $76.3(71.6)$ & & $55.8(39.1)$ & \\
\hline $\mathrm{T} 4$ & $62.5(52.1)$ & & $56.7(37.8)$ & \\
\hline Histological type & & 0.716 & & 0.470 \\
\hline $\begin{array}{l}\text { Invasive ductal } \\
\text { carcinoma }\end{array}$ & $80.0(73.8)$ & & $66.3(52.7)$ & \\
\hline Other types & $77.4(74.2)$ & & $70.6(60.5)$ & \\
\hline ER & & $<0.001$ & & $<0.001$ \\
\hline Negative & $70.0(60.4)$ & & $52.1(37.0)$ & \\
\hline Positive & $84.6(80.9)$ & & $75.2(62.7)$ & \\
\hline PR & & $<0.001$ & & $<0.001$ \\
\hline Negative & $69.0(61.3)$ & & $53.2(37.9)$ & \\
\hline Positive & $88.1(83.9)$ & & $78.4(66.8)$ & \\
\hline HER-2 & & 0.371 & & 0.421 \\
\hline Negative & $80.7(75.7)$ & & $70.0(54.1)$ & \\
\hline Positive & $76.4(69.1)$ & & $59.3(54.1)$ & \\
\hline Molecular subtype & & $<0.001$ & & $<0.001$ \\
\hline Luminal A & $98.4(96.3)$ & & 89.7 (77.2) & \\
\hline Luminal B & $75.5(71.1)$ & & $64.7(52.7)$ & \\
\hline
\end{tabular}

Table 2 (continued)
Table 2 (continued)

\begin{tabular}{|c|c|c|c|c|}
\hline Characteristic & $\begin{array}{c}3-y r(5-y r) \text { OS } \\
(\%)\end{array}$ & $P$ value & $\begin{array}{c}3-y r(5-y r) \text { DFS } \\
(\%)\end{array}$ & $P$ value \\
\hline $\begin{array}{l}\text { HER2- } \\
\text { overexpressing }\end{array}$ & $76.2(62.8)$ & & $53.2(53.2)$ & \\
\hline TNBC & $66.0(57.7)$ & & $52.1(26.9)$ & \\
\hline Ki67 & & 0.025 & & 0.016 \\
\hline$\leq 14 \%$ & $88.3(81.3)$ & & $76.1(64.3)$ & \\
\hline$>14 \%$ & 76.4 (71.3) & & $63.8(50.4)$ & \\
\hline \multicolumn{2}{|c|}{$\begin{array}{l}\text { The number of axillary involved } \\
\text { lymph nodes }\end{array}$} & $<0.001$ & & $<0.001$ \\
\hline 0 & $89.9(83.5)$ & & $79.4(66.8)$ & \\
\hline $1-3$ & $86.0(81.2)$ & & $72.8(65.7)$ & \\
\hline $4-9$ & $78.8(71.1)$ & & $60.5(46.0)$ & \\
\hline$\geq 10$ & $57.4(50.5)$ & & $46.2(29.4)$ & \\
\hline \multicolumn{2}{|c|}{$\begin{array}{l}\text { Involvement of infraclavicular } \\
\text { lymph node }\end{array}$} & $<0.001$ & & $<0.001$ \\
\hline No & $85.0(78.9)$ & & 72.5 (59.4) & \\
\hline Yes & $52.4(49.5)$ & & $40.0(27.5)$ & \\
\hline SCLD & & 0.018 & & 0.020 \\
\hline Yes & $69.8(65.8)$ & & $61.1(49.0)$ & \\
\hline No & $87.0(80.2)$ & & $72.0(58.3)$ & \\
\hline
\end{tabular}

ER, estrogen receptor; PR, progestational hormone; HER2 , human epidermal growth factor receptor-2; TNBC, triple negative breast cancer; SCLD, supraclavicular lymph node dissection.

the baseline of upfront independent prognostic factors were considered.

\section{Safety}

Among the 146 patients who underwent SCLD, 16 developed delayed swelling of the ipsilateral upper arm after surgery; the incidence was $10.96 \%$ (16/146). Postoperative cervical chylous lymphocele was noted in $6(4.1 \%)$ patients (24-hour volume of drainage greater than $200 \mathrm{~mL}$ ); they were managed by compression and drainage without the need for secondary surgery. None of the patients experienced postoperative supraclavicular hemorrhage, edema, movement disorders of the head and neck, pleural effusion, or chylothorax. 
Table 3 Multivariable analyses of factors associated with survival outcomes

\begin{tabular}{|c|c|c|c|c|}
\hline & \multicolumn{2}{|l|}{ OS } & \multicolumn{2}{|l|}{ DFS } \\
\hline ER (-) vs. ER (+) & * & 0.302 & $1.871(1.061-3.300)$ & 0.030 \\
\hline PR (-) vs. PR (+) & * & 0.215 & $1.826(1.032-3.232)$ & 0.039 \\
\hline \multicolumn{5}{|l|}{ Ki67 } \\
\hline Molecular subtype & & 0.000 & * & 0.227 \\
\hline Luminal B vs. Luminal A & $6.160(1.879-20.190)$ & 0.003 & * & 0.056 \\
\hline HER2-overexpressing vs. Luminal A & $11.562(3.119-42.857)$ & 0.000 & * & 0.931 \\
\hline TNBC vs. Luminal A & $12.091(3.623-40.351)$ & 0.000 & * & 0.561 \\
\hline N2 vs. no & $1.855(0.879-3.914)$ & 0.105 & $2.207(1.298-3.753)$ & 0.003 \\
\hline N3 vs. no & $3.202(1.489-6.888)$ & 0.003 & $3.331(1.905-5.824)$ & 0.000 \\
\hline \multicolumn{5}{|l|}{$\begin{array}{l}\text { Involvement of infraclavicular lymph } \\
\text { node }\end{array}$} \\
\hline No vs. yes & $0.544(0.305-0.973)$ & 0.040 & $0.568(0.354-0.913)$ & 0.019 \\
\hline \multicolumn{5}{|l|}{ SCLD } \\
\hline No vs. yes & * & 0.403 & * & 0.405 \\
\hline
\end{tabular}

*, HR $(95 \% \mathrm{Cl})$ was not given when P>0.05 in Cox proportional hazards regression analysis. ER, estrogen receptor; PR, progestational hormone; HER-2, human epidermal growth factor receptor-2; TNBC, triple negative breast cancer; SCLD, supraclavicular lymph node dissection.

\section{Discussion}

Patients with breast cancer who presented with ISLM at diagnosis, were previously considered to have a poor prognosis $(5,6)$. ISLM was therefore classified as stage M1 disease in the AJCC $5^{\text {th }}$ edition of 1987 . However, in 2001, Brito et al. (7) reported that patients with ISLM had better outcomes than patients with stage IV disease. Consequently, in 2003, the AJCC revised the TNM staging of breast cancer to categorize ISLM as stage $\mathrm{N} 3 \mathrm{c}$, clinical stage IIIC (any T, N3, M0). Therefore, breast cancer with ISLM should be regarded as locoregional disease and thus locoregional treatments such as regional RT or surgery may offer a better prognosis.

ISLM is known to confer a relatively poor prognosis in breast cancer. Several studies have reported the 5-year OS and DFS in patients with ISLM to range from $33.3 \%$ to $78 \%$ and $25 \%$ to $51 \%$, respectively $(7,12,15,17,18,20,21)$.
In the present study, the 3- and 5-year OS and DFS were $79.5 \%$ and $73.9 \%$, and $67.5 \%$ and $54.8 \%$, respectively. These findings were similar to that of previous studies. However, on univariate analyses of survival in either cohort, we found that patients with ISLM in the SCLD cohort had poor OS and DFS. We speculated that this difference in survival between the groups was probably caused by unbalanced baseline characteristics. So that we did not suggest unselected patients with ISLM accept SCLD as it was not beneficial for survival in the entire cohort of patients.

Locoregional RT after surgery for breast cancer with ISLM has provided acceptable in-field regional control rates in previous studies $(13,22)$. As opposed to radical RT, the role of SCLD remains controversial since ISLM is considered to be the precursor of distant spread, and local control is not a major factor. Chang et al. (15) suggested that SCLD should be considered in selected cases. Interestingly, 


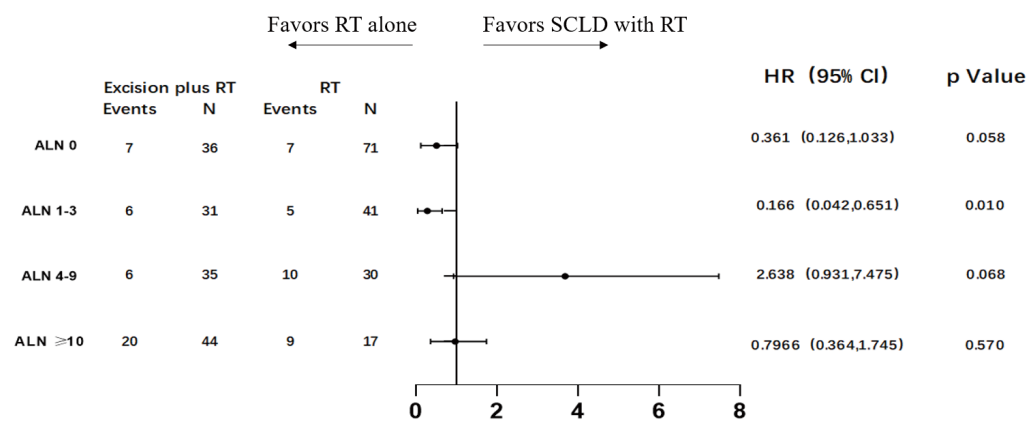

Figure 3 Forest plots of subgroup overall survival analyses stratifying by the number of involved axillary lymph nodes.

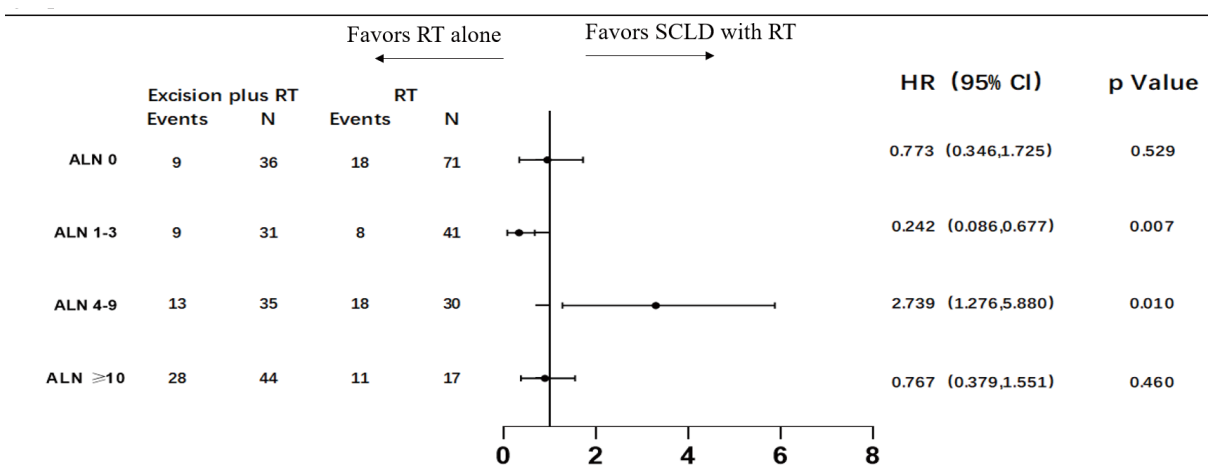

Figure 4 Forest plots of subgroup disease-free survival analyses stratifying by the number of involved axillary lymph nodes.

Jung et al. (17) reported that on univariate analysis, local aggressive treatments, including SCLD, significantly improved OS. In an interesting study on practices for the management of breast cancer metastases to the neck in the UK (23), the majority of breast surgeons preferred not to perform neck dissection in patients with ISLM from breast cancer. Conversely, most otolaryngologists, oral and maxillofacial surgeons, and head and neck oncologists expressed opposing views. This probably suggests that breast surgeons, being trained in another specialty, do not prefer neck dissection. In view of these reports, further investigation is needed into the possible benefits of SCLD in breast cancer.

In this study, we also compared the prognosis of different modalities of local therapy by performing additional stratified analyses between the groups. This was performed to identify a subgroup of patients with ISLM who may benefit from SCLD. In either group, all conclusions were based on balanced baseline independent prognostic factors. After stratification based on the number of involved axillary nodes, no significant differences in OS and DFS were noted between patients with 0 and more than 9 positive axillary lymph nodes in either group. This probably suggests that in patients with ISLM who have no positive axillary lymph nodes after NAC, local RT and systemic treatment are adequate for achieving a good prognosis, and SCLD is unnecessary. In theory, a larger tumor load after NAC in patients with ISLM predisposes to distant metastasis; in patients with ISLM and more than 9 positive axillary lymph nodes after NAC, distant micrometastasis are very likely to have occurred, and circulating tumor cells or cancer stem cells may persist. Therefore, local therapy may not be particularly effective. We were unable to draw conclusions in the subgroup of patients with 1-3 and 4-9 positive axillary lymph nodes owing to imbalances of other independent survival factors at baseline. On further investigation, we identified a subgroup of patients with ISLM who had non-luminal A tumors and 4-9 positive axillary lymph nodes; this subgroup experienced better OS and DFS after SCLD when the 2 independent prognostic factors were combined. It is possible that patients in this subgroup obtained no additional benefit from endocrine 


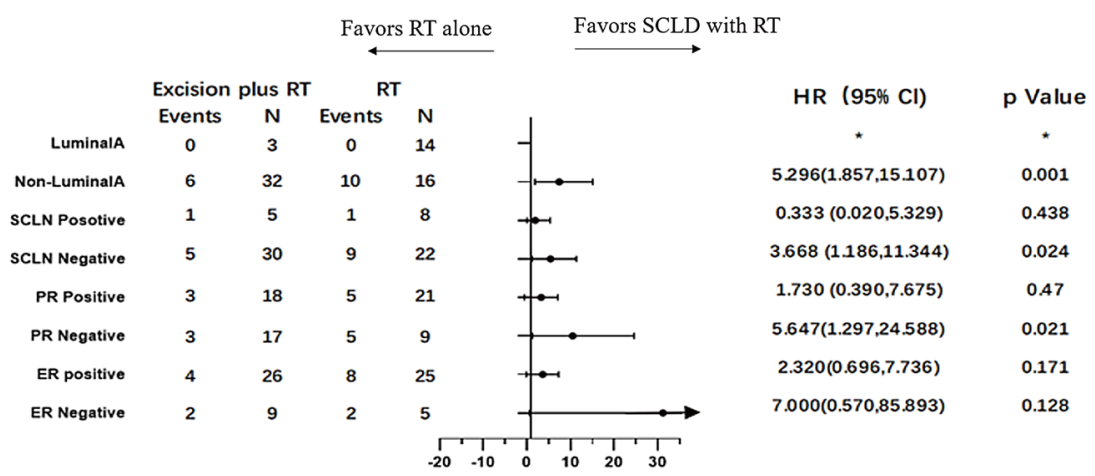

Figure 5 Forest plots of subgroup overall survival analyses of 4-9 positive axillary lymph nodes involved.
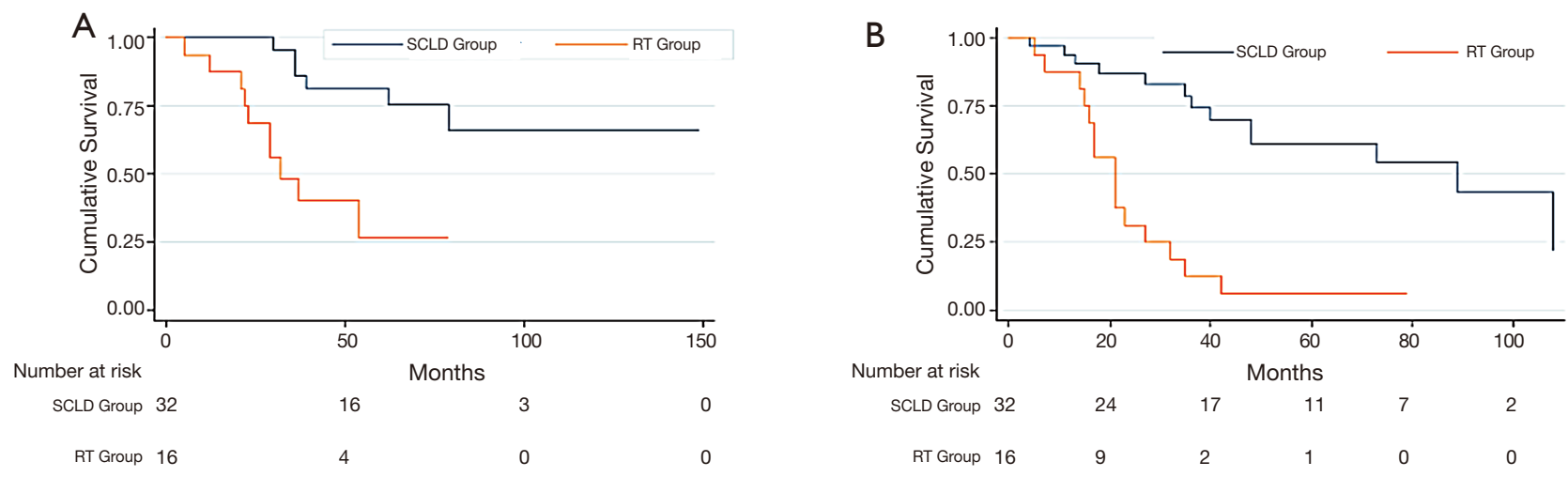

Figure 6 Kaplan-Meier analysis in patients with non-luminal A tumors and 4-9 positive axillary lymph nodes. (A) Kaplan-Meier analysis comparing overall survival in patients with non-luminal A tumors and 4-9 positive axillary lymph nodes treated with SCLD plus RT and RT alone patients; (B) Kaplan-Meier analysis comparing disease-free survival in patients with non-luminal A tumors and 4-9 positive axillary lymph nodes treated with SCLD plus RT and RT alone patients. SCLD, supraclavicular lymph node dissection; RT, radiotherapy.

therapy. In addition, patients with luminal A breast cancer have been reported to obtain greater benefit from regional RT $(24,25)$. Therefore, SCLD was probably unable to improve the prognosis further. Local radical treatment may therefore offer greater benefits in patients with non-luminal A tumors and 4-9 positive axillary lymph nodes. However, from the data in the present cohort, it was not possible to determine whether SCLD may offer benefits to patients with ISLM and 1-3 positive axillary lymph nodes.

This study is probably the first to suggest that in patients with ISLM, SCLD may be particularly beneficial to those with non-luminal A tumors and 4-9 positive axillary lymph nodes; it offers better local control, reduces distant metastases, and prolongs survival. Interestingly, Zhang et al. (26) had reported that SCLD may be effective in improving local control in patients with ISLM, particularly in those with ER and PR negative tumors. This concurs with our findings to a certain extent. As noted from the findings of the present study, SCLD may be unnecessary in patients with ISLM and 0 or more than 9 positive axillary lymph nodes after NAC.

Since all the patients included in this study were diagnosed with locally advanced breast cancer, the regional lymph nodes were likely to be in close proximity to the peripheral blood vessels and connective tissue. NAC was therefore employed to reduce the size of the regional lymph nodes, which were significantly reduced or clearly separated from the peripheral blood vessels, thereby reducing the risks during surgery.

Our study has some limitations. Firstly, a pathologic diagnosis of ISLM was not available; patients were clinically 


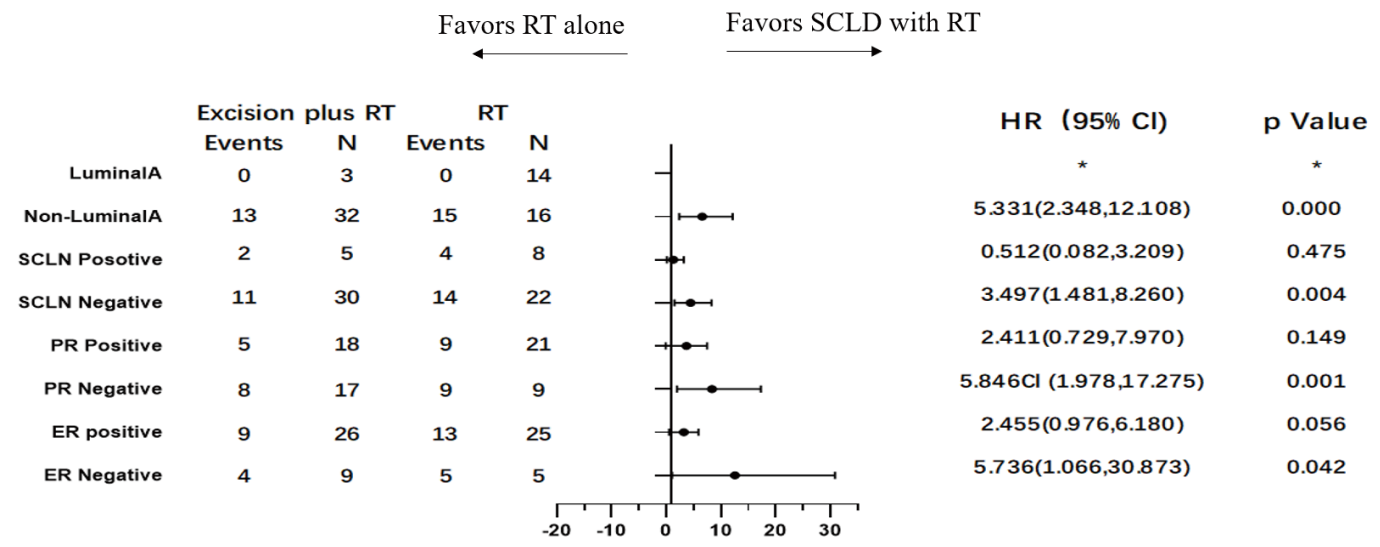

Figure 7 Forest plots of subgroup disease-free survival analyses of 4-9 positive axillary lymph nodes involved.

staged based on the typical findings on lymph node imaging. Fine-needle aspiration of the supraclavicular lymph nodes was not routinely performed at our institute during the study period. Secondly, the sample size was inadequate and potential selection bias selection bias still existed. However, it has the largest sample size among all current studies. Despite these limitations, on stratified analysis, we identified a subgroup of patients who could benefit from SCLD. Further detailed stratification requires a larger sample size for the accurate identification of patients who may benefit from SCLD. However, one of the unique strengths of this study is that a large homogeneous cohort was available from a single institution.

The implementation of SCLD requires screening to identify appropriate patients. An accurate preoperative assessment of cervical lymph nodes and clinicopathologic features provides an important basis for decision-making in SCLD. The scope of SCLD should be standardized on the basis of the AJCC $8^{\text {th }}$ edition; dissection should be performed in the anatomical extent of the supraclavicular lymph nodes, and the lymph nodes and tissues in this area should be thoroughly cleared with regard to the anatomy of the fascial planes. Attention should be paid to the management of lymph nodes in the angle of the jugular vein, which is often the site of recurrence and metastasis. The surgical results in our institute were acceptable, with a very low incidence of surgical complications, which were easily managed.

\section{Conclusions}

Synchronous ISLM in breast cancer should be considered as locoregional disease, and should be treated with a curative intent. Comprehensive treatment including systemic therapy, surgery, and RT should be offered to patients with ISLM, and SCLD should be considered as part of the multimodality treatment approach in patients with nonluminal A tumors and 4-9 positive axillary lymph nodes. Highly individualized comprehensive treatment is strongly recommended for patients with ISLM from breast cancer. Further prospective studies should be performed with larger samples to further confirm the value of SCLD in selected patients with ISLM.

\section{Acknowledgments}

Funding: None.

\section{Footnote}

Conflicts of Interest: All authors have completed the ICMJE uniform disclosure form (available at http://dx.doi. org/10.21037/gs.2020.03.09). The authors have no conflicts of interest to declare.

Ethical Statement: The authors are accountable for all aspects of the work in ensuring that questions related to the accuracy or integrity of any part of the work are appropriately investigated and resolved. The study was approved by the Ethics Committee of the First Affiliated Hospital of Army Medical University, PLA.

Open Access Statement: This is an Open Access article distributed in accordance with the Creative Commons 
Attribution-NonCommercial-NoDerivs 4.0 International License (CC BY-NC-ND 4.0), which permits the noncommercial replication and distribution of the article with the strict proviso that no changes or edits are made and the original work is properly cited (including links to both the formal publication through the relevant DOI and the license). See: https://creativecommons.org/licenses/by-nc-nd/4.0/.

\section{References}

1. Galper S, Recht A, Silver B, et al. Factors associated with regional nodal failure in patients with early stage breast cancer with 0-3 positive axillary nodes following tangential irradiation alone. Int J Radiat Oncol Biol Phys 1999;45:1157-66.

2. Recht A, Gray R, Davidson NE, et al. Locoregional failure 10 years after mastectomy and adjuvant chemotherapy with or without tamoxifen without irradiation: experience of the Eastern Cooperative Oncology Group. J Clin Oncol 1999;17:1689-700.

3. Chen SC, Chen MF, Hwang TL, et al. Prediction of supraclavicular lymph node metastasis in breast carcinoma. Int J Radiat Oncol Biol Phys 2002;52:614-9.

4. American Joint Committee on Cancer. Manual for Staging of Cancer, 5th ed. Philadelphia: JB Lippincott, 1997.

5. Kiricuta IC, Willner J, Kolbl O, et al. The prognostic significance of the supraclavicular lymph node metastases in breast cancer patients. Int J Radiat Oncol Biol Phys 1994:28:387-93.

6. Debois JM. The significance of a supraclavicular node metastasis in patients with breast cancer. A literature review. Strahlenther Onkol 1997;173:1-12.

7. Brito RA, Valero V, Buzdar AU, et al. Long-term results of combined-modality therapy for locally advanced breast cancer with ipsilateral supraclavicular metastases: The University of Texas M.D. Anderson Cancer Center experience. J Clin Oncol 2001;19:628-33.

8. Singletary SE, Allred C, Ashley P, et al. Revision of the American Joint Committee on Cancer staging system for breast cancer. J Clin Oncol 2002;20:3628-36.

9. Liu XH, Zhang L, Chen B. A meta-analysis of the prognosis in patients with breast cancer with ipsilateral supraclavicular lymph node metastasis versus patients with stage IIIb/c or IV breast cancer. Chronic Dis Transl Med 2016;1:236-42.

10. Deo SV, Purkayastha J, Shukla NK, et al. Intent of therapy in metastatic breast cancer with isolated ipsilateral supraclavicular lymph node spread--a therapeutic dilemma. J Assoc Physicians India 2003;51:272-5.

11. Huang EH, Strom EA, Valero V, et al. Locoregional treatment outcomes for breast cancer patients with ipsilateral supraclavicular metastases at diagnosis. Int J Radiat Oncol Biol Phys 2007;67:490-6.

12. Park HJ, Shin KH, Cho KH, et al. Outcomes of positron emission tomography-staged clinical N3 breast cancer treated with neoadjuvant chemotherapy, surgery, and radiotherapy. Int J Radiat Oncol Biol Phys 2011;81:e689-95.

13. Kim K, Jeong Y, Shin KH, et al. Impact of Regional Nodal Irradiation for Breast Cancer Patients with Supraclavicular and/or Internal Mammary Lymph Node Involvement: A Multicenter, Retrospective Study (KROG 16-14). Cancer Res Treat 2019;51:1500-8.

14. Wu SG, Sun JY, Zhou J, et al. The value of radiotherapy in breast cancer patients with isolated ipsilateral supraclavicular lymph node metastasis without distant metastases at diagnosis: a retrospective analysis of Chinese patients. Onco Targets Ther 2014;7:281-8.

15. Chang XZ, Yin J, Sun J, et al. A retrospective study of different local treatments in breast cancer patients with synchronous ipsilateral supraclavicular lymph node metastasis. J Cancer Res Ther 2013;9 Suppl:S158-61.

16. Chen SC, Chang HK, Lin YC, et al. Prognosis of breast cancer after supraclavicular lymph node metastasis: not a distant metastasis. Ann Surg Oncol 2006;13:1457-65.

17. Jung J, Kim SS, Ahn SD, et al. Treatment Outcome of Breast Cancer with Pathologically Proven Synchronous Ipsilateral Supraclavicular Lymph Node Metastases. J Breast Cancer 2015;18:167-72.

18. Noh JM, Kim KH, Park W, et al. Prognostic significance of nodal involvement region in clinical stage IIIc breast cancer patients who received primary systemic treatment, surgery, and radiotherapy. Breast 2015;24:637-41.

19. Fan $Y, X u B$, Liao $Y$, et al. A retrospective study of metachronous and synchronous ipsilateral supraclavicular lymph node metastases in breast cancer patients. Breast 2010;19:365-9.

20. Olivotto IA, Chua B, Allan SJ, et al. Long-term survival of patients with supraclavicular metastases at diagnosis of breast cancer. J Clin Oncol 2003;21:851-4.

21. Dienstmann R, Branco LG, Rezende LM, et al. Combined modality therapy of stage IIIC breast cancer. Breast J 
2011;17:331-3.

22. Poortmans PM, Collette S, Kirkove C, et al. Internal Mammary and Medial Supraclavicular Irradiation in Breast Cancer. N Engl J Med 2015;373:317-27.

23. Bisase B, Kerawala C. Survey of UK practice for management of breast cancer metastases to the neck. Ann R Coll Surg Engl 2012;94:484-9.

24. Mao JH, Diest PJV, Perez-Losada J, et al. Revisiting the impact of age and molecular subtype on overall survival after radiotherapy in breast cancer patients. Sci Rep 2017;7:12587.

Cite this article as: Ai X, Wang M, Li J, Hu Y, Hou L, Zheng X, Yan Y, Pan Q, Jin Y, Liu W, Tan X, Tian Y, Zhang Y, Tang P, Jiang J. Supraclavicular lymph node dissection with radiotherapy versus radiotherapy alone for operable breast cancer with synchronous ipsilateral supraclavicular lymph node metastases: a real-world cohort study. Gland Surg 2020;9(2):329341. doi: $10.21037 /$ gs.2020.03.09
25. Lim YJ, Lee SW, Choi N, et al. Failure patterns according to molecular subtype in patients with invasive breast cancer following postoperative adjuvant radiotherapy: long-term outcomes in contemporary clinical practice. Breast Cancer Res Treat 2017;163:555-63.

26. Zhang W, Qi XM, Chen AX, et al. [The Role of Supraclavicular lymph node dissection in Breast Cancer Patients with Synchronous Ipsilateral Supraclavicular Lymph Node Metastasis]. Zhonghua Zhong Liu Za Zhi 2017;39:374-9. 
Supplementary

Table S1 SCLD group vs. RT group clinicopathological characteristics

\begin{tabular}{|c|c|c|c|}
\hline Characteristics & $\operatorname{SCLD}(n=146)$ & $\mathrm{RT}(\mathrm{n}=159)$ & $P$ value \\
\hline \multicolumn{4}{|l|}{ Age (years) } \\
\hline$<35$ & 11 & 11 & \\
\hline$\geq 35$ & 135 & 148 & 0.835 \\
\hline \multicolumn{4}{|l|}{ Tumor location } \\
\hline Left & 87 & 93 & \\
\hline Right & 59 & 66 & 0.846 \\
\hline \multicolumn{4}{|l|}{ Tumor quadrant } \\
\hline Outer upper & 92 & 94 & \\
\hline Outer lower & 12 & 15 & \\
\hline Inner upper & 13 & 15 & \\
\hline Inner lower & 6 & 11 & \\
\hline central & 23 & 24 & 0.838 \\
\hline \multicolumn{4}{|l|}{ Primary tumor size } \\
\hline $\mathrm{T} 1$ & 20 & 36 & \\
\hline $\mathrm{T} 2$ & 94 & 101 & \\
\hline T3 & 17 & 12 & \\
\hline $\mathrm{T} 4$ & 15 & 8 & 0.067 \\
\hline \multicolumn{4}{|l|}{ Histological type } \\
\hline Invasive ductal carcinoma & 121 & 130 & \\
\hline Other types & 25 & 29 & 0.799 \\
\hline \multicolumn{4}{|l|}{ ER } \\
\hline Negative & 59 & 48 & \\
\hline Positive & 87 & 111 & 0.062 \\
\hline \multicolumn{4}{|l|}{ PR } \\
\hline Negative & 77 & 62 & \\
\hline Positive & 69 & 97 & 0.016 \\
\hline \multicolumn{4}{|l|}{ HER-2 } \\
\hline Negative & 110 & 113 & \\
\hline Positive & 36 & 46 & 0.337 \\
\hline \multicolumn{4}{|l|}{ Molecular subtype } \\
\hline Luminal A & 19 & 61 & \\
\hline Luminal B & 71 & 50 & \\
\hline HER-2 overexpressing & 21 & 19 & \\
\hline TNBC & 35 & 29 & $<0.001$ \\
\hline \multicolumn{4}{|l|}{ Ki67 } \\
\hline$\leq 14 \%$ & 31 & 51 & \\
\hline$>14 \%$ & 115 & 108 & 0.033 \\
\hline \multicolumn{4}{|c|}{ The number of involved axillary lymph nodes } \\
\hline 0 & 36 & 71 & \\
\hline $1-3$ & 31 & 41 & \\
\hline $4-9$ & 35 & 30 & \\
\hline$\geq 10$ & 44 & 17 & $<0.001$ \\
\hline \multicolumn{4}{|c|}{ Involvement of infraclavicular lymph node } \\
\hline No & 110 & 141 & \\
\hline Yes & 36 & 18 & 0.002 \\
\hline \multicolumn{4}{|l|}{ NAC } \\
\hline$P D+S D$ & 50 & 69 & \\
\hline $\mathrm{PR}+\mathrm{CR}$ & 96 & 90 & 0.089 \\
\hline
\end{tabular}

SCLD, supraclavicular lymph node dissection; RT, radiotherapy; ER, estrogen receptor; PR, progestational hormone; HER2, human epidermal growth factor receptor-2; TNBC, triple negative breast cancer; NAC, neoadjuvant chemotherapy; PD, progressive disease; SD, stable disease; PR, partial disease; CR, complete disease. 